Stimuli bewirken einen starken Dopamin-Opiat-Kick - der dank Internet jederzeit schnell verfügbar ist. Cybersex wird daher auch „das Crack der Sexsucht“ genannt. Wenn das opiatgestützte Genussempfinden mit der Zeit abnimmt, kommt es zum dopaminbedingten Craving nach stärkeren Reizen - und damit zum Kontrollverlust.

Exzessiver Konsum von InternetPornografie ist inzwischen auch bei Jugendlichen stark verbreitet. Darauf machte die Psychologin Tabea Freitag aus Haste aufmerksam. Nach Studien des KFN sehen $8 \%$ der 15-jährigen Jungen und $16 \%$ der männlichen Studenten täglich Pornofilme. Nicht selten sind darunter auch Videos mit illegalen Inhalten wie Vergewaltigungen oder Sex mit Kindern. Die Einschätzung, Jugendliche „könnten ja zwischen Fiktion und Realität unterscheiden“, hält Freitag daher auch für unsinnig. Zum einen steckt hinter den Sexfilmen eine sehr bittere Realität, zum anderen belegen Studien, dass die Wertvorstellungen der jugendlichen Voyeure durch die Bilder geprägt werden: Je häufiger Jugendliche in den einschlägigen Internetportalen unterwegs sind, desto realistischer und „nützlicher" für ihr Sexualleben erscheint ihnen das Gesehene. Dadurch sinkt außer der Zufriedenheit mit der eigenen Sexualität auch die Empathie- und Bindungsfähigkeit. Gleichzeitig verstärkt exzessiver Pornografiekonsum die Neigung, deviante Sexualpraktiken und sexuelle Gewalt zu tolerieren und auch selbst anzuwenden.

\section{Neunjähriger Nachahmungstäter}

Freitag schilderte den Fall eines Neunjährigen, bei dem die Eltern im Browserverlauf Links wie „heiße Thai-Girls“, und „der härteste Sex“ entdeckten, die von einem Mitschüler an die ganze Klasse weitergeleitet worden waren. Obwohl die Eltern gut damit umgingen und offen mit dem Jungen darüber sprachen, verschaffte er sich immer wieder Zugang zu pornografischen Darstellungen. Wenig später wurde er beim sexuellen Missbrauch seiner Schwester erwischt.

Um Nachahmertaten und Abhängigkeit zu verhindern, hält Freitag präventive Maßnahmen für extrem wichtig. „Wir müssen uns daran gewöhnen, unsere Patienten immer auch nach dem Gebrauch von Internetpornografie zu fragen“, so Freitag. In Präventionsveranstaltungen hat sie die Erfahrung gemacht, dass viele Jugendliche sich sogar wünschen, mit jemanden über ihre ambivalenten Erfahrungen mit Pornografie
- zwischen Faszination und Abgestoßensein - reden zu können.

\section{Medienkompetenz durch Medienabstinenz}

Wie vermittelt man Kindern einen souveränen Umgang mit Computer und Internet? Die Annahme, dass Kinder sich schon möglichst früh mit den später unverzichtbaren Medien auseinandersetzen sollten, hält Möller für gänzlich falsch. Seine Überzeugung ist: „Medienkompetenz beginnt mit Medienabstinenz." Kinderzimmer sollten bildschirmfrei sein. „Ein eigener PC ist oft der Einstieg in ein problematisches Verhalten“, so Möller. Kinder müssen die Möglichkeit haben, umfassende Sinneserfahrungen zu machen, indem sie frei spielen, sich bewegen, vorgelesen bekommen - anstatt nur von visuellen und auditiven Reizen überflutet $\mathrm{zu}$ werden. Fröhliche, aktive, interessierte Kinder könnten selbstbestimmt mit dem Computer umgehen, betont Möller. Dabei sei eine kritische Begleitung durch die Eltern sinnvoll, etwa wenn es um private Daten in sozialen Netzwerken wie Facebook geht.

DR. BEATE SCHUMACHER

- 13. Interdisziplinärer Kongress für Suchtmedizin, München, 5.-7. Juli 2012

\title{
Wege aus der Internetsucht
}

Bisher gibt es nur wenige Behandlungsangebote für nicht stoffgebundene Abhängigkeitserkrankungen wie die Internetsucht. Das Kinderkrankenhaus auf der Bult hat seit 2010 sechs Behandlungsplätze für internet- und computersüchtige Jugendliche. Im Zentrum der Therapie steht die Behandlung der Grundstörung. Durch Einzel- und Familientherapie soll den Patienten eine Perspektive jenseits des Bildschirmhorizonts vermittelt werden. Außerdem lernen die Jugendlichen, wieder eine klare Tagesstruktur einzuhalten und im sozialen Miteinander zurechtzukommen. Auch der kontrollierte Umgang mit Medien wird geübt.

Eine ambulante Gruppentherapie für Jugendliche und Erwachsene mit Internetsucht wird seit 2008 von der Ambulanz für Spielsucht an der Universitätsklinik Mainz angeboten. Wie

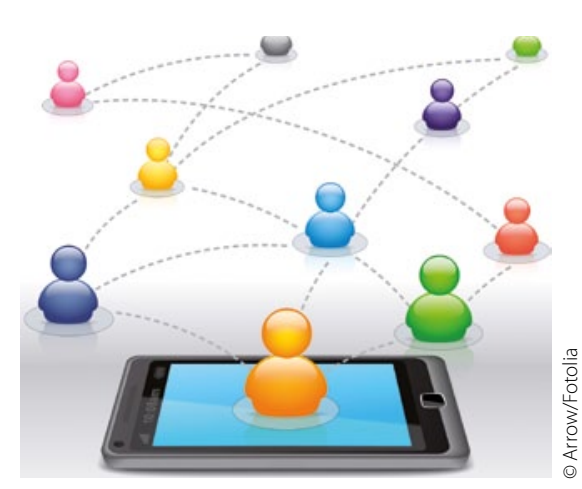

Einsam im sozialen Netz. der psychologische Leiter Dr. Klaus Wölfling berichtet, liegt der Altersgipfel der zumeist männlichen Patienten zwischen 16 und 22 Jahren. Für die Behandlung wurden kognitiv-verhaltenstherapeutische Ansätze zur Therapie stoffbezogener Süchte adaptiert. Im Vordergrund steht die individuelle Analyse des Suchtverhaltens. In Wochenprotokollen dokumentieren die Patienten, welche Situationen das Suchtverhalten auslösen. Vorteil der ambulanten Umgebung ist, dass die „normalen“ Lebensbedingungen und damit zusammenhängende Rückfälle mit in die Behandlung einbezogen werden können. Hauptziel der Intervention ist die Abstinenz von dysfunktionaler Internetnutzung. Die gelang in einer Pilotstudie sechs Monate nach der Therapie noch etwa der Hälfte der Patienten. Was die Intervention langfristig bringt, soll jetzt in einer deutschen Multicenter-Studie überprüft werden. 